\section{Global Stabilization Via Local Stabilizing Actions}

\author{
A. Bülent Özgüler
}

\begin{abstract}
Stabilization of a linear, time-invariant system via stabilization of its main diagonal subsytems is the underlying problem in all diagonal dominance techniques for decentralized control. In these techniques as well as all Nyquist-based techniques, sufficient conditions are obtained under the assumption that the collection of the unstable poles of all diagonal subsystems is the same as the unstable poles of the overall system. We show that this assumption is by itself enough to construct a solution to the problem at least in cases where the diagonal subsystems have disjoint poles.
\end{abstract}

Index Terms-Decentralized control, diagonal dominance, interconnected systems, stabilization.

\section{INTRODUCTION}

Consider an $N$-channel $p \times m$ multivariable system in transfer matrix representation

$$
Z(s)=\left[\begin{array}{ccc}
Z_{11}(s) & \cdots & Z_{1 N}(s) \\
\vdots & & \vdots \\
Z_{N 1}(s) & \cdots & Z_{N N}(s)
\end{array}\right]
$$

where $Z_{i j}(s)$ is $p_{i} \times m_{j}$ for $i, j \in \mathbf{N}:=\{1, \ldots, N\}$ with $p=\sum_{i} p_{i}, m=\sum_{j} m_{j}$. A main problem in decentralized control is to determine whether the $p \times m$ system can be stabilized by local controllers $Z_{c i}(s), i \in \mathbf{N}$, each of size $m_{i} \times p_{i}$ and each stabilizing its own main diagonal subsystem represented by $Z_{i i}(s)$. Alternatively, the problem is finding a decentralized controller $Z_{c}=\operatorname{diag}\left\{Z_{c 1}, Z_{c 2}, \ldots, Z_{c N}\right\}$ simultaneously stabilizing $Z$ and its diagonal part $Z_{d}:=\operatorname{diag}\left\{Z_{11}, Z_{22}, \ldots, Z_{N N}\right\}$, [5]. This is a fundamental problem of decentralized action since it seeks an answer to the question "When do local solutions result in a similar global solution?"

The problem can be traced to the work of Rosenbrock [6], where well-known single-input-single-output (SISO) frequency domain design techniques are extended to multiloop systems satisfying certain "diagonal dominance" or "weak interaction" properties. A review of the existing results on the problem can be found in [2, Ch. 4]. In [15] and [3], related problems are studied. In [9], the problem for $N=2$ has been posed as one of reliable stabilization of a feedforward interconnected system. In [10], a multichannel generalization of the problem has been posed as a decentralized concurrent stabilization problem and it has been established that the problem is equivalent to decentralized strong stabilization of a transformed plant resulting from an application of an initial decentralized controller to $Z$. The so called "decentralized blocking zeros" are in turn central to the solution of the decentralized strong stabilization problem, [10].

Let $\mathbf{P}$ denote the set of (proper) transfer functions and let $G(s) \in \mathbf{P}^{p \times m}$ be a transfer matrix. A number $s_{0}$ in the extended closed right half complex plane is called an unstable blocking zero of $G(s)$ if $G\left(s_{0}\right)=0$. Similarly, $s_{0}$ is called an unstable decentralized blocking zero of $Z$ if for some permutation $\left\{i_{1}, \ldots, i_{N}\right\}$ of $\mathbf{N}$ the following holds: $Z_{i_{k} i_{l}}\left(s_{0}\right)=0, k=1, \ldots, N, l=1, \ldots, k$. A main result of [10] and [8] on decentralized strong stabilization problem is that $Z$ admits a stable decentralized stabilizing controller if and only

Manuscript received November 29, 2004; revised July 18, 2005 and October 12, 2005. Recommended by Associate Editor U. Jonsson.

The author is with the Department of Electrical and Electronics Engineering, Bilkent University, Ankara 06800 Turkey (e-mail: ozguler@ee.bilkent.edu.tr).

Digital Object Identifier 10.1109/TAC.2005.864201 if there are an even number of poles of $Z$ between evey pair of real unstable decentralized blocking zeros of $Z$. The constructive part of the proof of this result requires the assumptions

$$
\begin{aligned}
& Z \text { is strongly connected, [1], and } \quad \forall i, j \in \mathbf{N}, i \neq j \\
& \operatorname{rank} Z_{i j} \geq 2 \text { or rank } Z_{j i} \geq 2 .
\end{aligned}
$$

The problem of simultaneously stabilizing $Z$ and $Z_{d}$ is equivalent to decentralized strong stabilization of some initially compensated system $\bar{Z}\left(Z_{c 0}\right)$, where $Z_{c 0}=\operatorname{diag}\left\{Z_{c 01}, \ldots, Z_{c 0 N}\right\}$ is a stabilizing controller for $Z_{d}$. While the decentralized blocking zeros of $\bar{Z}\left(Z_{c 0}\right)$ can be explicitly described independent of the initial controller $Z_{c 0}$, its poles depend on the choice of $Z_{c 0}$. This result of [10] is thus not as transparent as one would desire. Nevertheless, there are three important special cases, where the condition for solvability can be stated purely on the original plant [8]. The difference plant $Z_{\text {off }}:=Z-Z_{d}$ plays a major role in all these cases. This is natural to expect since our problem is one of simultaneous stabilization using a special structure controller and since, by [12, Lemma 5.4.20], some parity interlacing property of the difference plant is the main solvability condition for simultaneous stabilization of two plants.

i) In case the difference plant $Z_{\text {off }}$ is stable, under any diagonally stabilizing initial controller $Z_{c 0}$, the plant $\bar{Z}\left(Z_{c 0}\right)$ can be shown to be decentrally strong stabilizable. What makes the problem nontrivial is thus the unstable poles of the difference plant.

ii) Suppose that the diagonal subplants $Z_{i i}$ are all stable. The problem has a solution if and only if $Z_{\text {off }}$ is strong decentralized stabilizable, i.e., there are an even number of its poles between each pair of its real unstable decentralized blocking zeros.

iii) Suppose a minimal realization of $Z$ is stabilizable and detectable from every channel $i=1, \ldots, N$. If $N$ is odd, then $Z$ and $Z_{d}$ can always be simultaneously stabilized by a decentralized controller. If $N$ is even, then $Z$ and $Z_{d}$ can be simultaneously stabilized if and only if there are an even number of real poles of $Z$, counted with multiplicities, between each pair of real unstable decentralized blocking zeros of $Z_{\text {off }}$.

Note, with regard to iii), that, by definition, stabilizability and detectability from any one of the channels, say channel-1, would actually be sufficient to stabilize the overall system by a local stabilizing controller applied there. The diagonal subsystems of the resulting closed loop system would be also all stable. This would not however constitute a solution to our problem which assumes that the local controllers at channels $2, \ldots, N$ are "blind" to what goes on in channel-1. The local actions at channels $2, \ldots, N$ to stabilize the respective subsystems would hence, in general, destroy the stabilizing action taken by the local controller at channel-1.

We investigate, in the next section, another case for which the decentralized simultaneous stabilization of $Z$ and $Z_{d}$ is made possible by an assumption on $Z_{\text {off }}$. The main result, Theorem 2, can be obtained by investigating the decentralized strong stabilizability of $\bar{Z}\left(Z_{c 0}\right)$. However, we will give a direct proof, thereby eliminating the connectivity assumptions (2). We also focus on the case $N=2$ and state and prove the results for the two-channel case only for notational clarity. All results of the next section, Lemma 1, and Theorems 1 and 2, are valid in the $N$-channel case, but details have to be worked out.

\section{Diagonal Dominance Methods}

All Nyquist array based (block) diagonal dominance methods to decentralized control, [14], and many of the "interaction measure" [2] techniques are based on the following assumption. 
(A) $Z$ and $Z_{d}=\operatorname{diag}\left\{Z_{11}, \ldots, Z_{N N}\right\}$ have the same number of unstable poles with multiplicities.

The assumption clearly concerns the difference plant $Z_{\text {off }}$ and one expects that simultaneous stabilization problem will be easier to solve under such an assumption. However, the relevance of $\mathbf{A}$ to decentralized stabilization needs clarification. For instance, [11], it is neither implied by nor implies the lack of unstable decentralized fixed modes: Consider $Z=\left[Z_{i j}\right], i, j=1,2$ with $Z_{11}=(1) /(s-1), Z_{12}=$ $1, Z_{21}=(s) /\left((s-1)^{2}\right), Z_{22}=(1) /(s-1)$. Assumption $\mathbf{A}$ is satisfied for $Z$ since both $Z$ and $\operatorname{diag}\left\{Z_{11}, Z_{22}\right\}$ have a double pole at $s=1$. However, $Z$ has an unstable decentralized fixed mode at $s=1$. Also, a plant can be devoid of unstable decentralized fixed modes while A fails. Let $Z_{11}=Z_{12}=Z_{22}=(1) /(s+1), Z_{21}=(1) /(s)$. The $2 \times 2$ plant $Z=\left[Z_{i j}\right]$ has no unstable decentralized fixed modes and A fails.

We clarify, in Theorem 1, the connection between $\mathbf{A}$ and unstable decentralized fixed modes in an important special case. We first need the following result in which $\mathbf{S}$ denotes the set of stable transfer functions.

Lemma 1: If $\mathbf{A}$ holds and if the unstable poles of the diagonal subsystems $Z_{11}$ and $Z_{22}$ are disjoint, then the overall transfer matrix $Z$ has the bicoprime representation $Z=$

$$
\left[\begin{array}{l}
P_{11} C_{1} \\
P_{22} C_{2}
\end{array}\right]\left(D_{1} D_{22} C_{22} C_{1}\right)^{-1}\left[\begin{array}{ll}
D_{1} R_{11} & D_{2} R_{22}
\end{array}\right]
$$

for some nonsingular matrices $D_{i}, C_{i}, D_{i i}, C_{i i}$ and matrices $P_{i i}, R_{i i}, i=1,2$ over $\mathbf{S}$ satisfying $D_{1} D_{22}=D_{2} D_{11}, C_{22} C_{1}=$ $C_{11} C_{2}$ and such that the following fractions are coprime:

$$
P_{11} C_{22}^{-1}, D_{11}^{-1} R_{22}, P_{22} C_{11}^{-1}, D_{22}^{-1} R_{11} .
$$

Proof: Consider an arbitrary bicoprime fractional representation $Z=\left[\begin{array}{ll}P_{1}^{\prime} & P_{2}^{\prime}\end{array}\right]^{\prime} Q^{-1}\left[\begin{array}{ll}R_{1} & R_{2}\end{array}\right]$, where 'prime' denotes "transpose," over $\mathbf{S}$ in which, say, $Q$ is $r \times r$. Let $C_{i}=\operatorname{gcrf}\left\{P_{i}, Q\right\}$ and write $P_{i}=P_{i i} C_{i}, Q=\bar{Q}_{i} C_{i}$ for $i=1,2$. Also let $D_{i}=\operatorname{gclf}\left\{\bar{Q}_{i}, R_{i}\right\}$ and write $\bar{Q}_{i}=D_{i} Q_{i}, R_{i}=D_{i} R_{i i}$ for $i=1,2$. Here, "gcr(1)f" denotes "greatest common right (left) factor" so that the matrices $\left(\bar{Q}_{i}, P_{i i}\right)$ are right coprime and $\left(Q_{i}, R_{i i}\right)$ are left coprime over $\mathbf{S}$, see [4]. Since we started out with a bicoprime representation, $\left(D_{1}, D_{2}\right)$ is left coprime, $\left(C_{2}, C_{1}\right)$ are right coprime, and we write $D_{1}^{-1} D_{2}=D_{22} D_{11}^{-1}, C_{2} C_{1}^{-1}=C_{11}^{-1} C_{22}$ for right coprime $\left(D_{22}, D_{11}\right)$ and for left coprime $\left(C_{11}, C_{22}\right)$ over $\mathbf{S}$. It follows that

$$
\operatorname{det} C_{i} \simeq \operatorname{det} C_{i i}, \operatorname{det} D_{i} \simeq \operatorname{det} D_{i i}, \quad i=1,2
$$

where, for $a, b \in \mathbf{S}, a \simeq b$ means that $a$ and $b$ are associates, i.e., they are equal upto multiplication by a unit of $\mathbf{S}$. Noting that $Q=$ $D_{1} Q_{1} C_{1}=D_{2} Q_{2} C_{2}$, we can then write

$$
C_{22} Q_{1}^{-1} D_{22}=C_{11} Q_{2}^{-1} D_{11}
$$

Now, the diagonal subsytem transfer matrices are $Z_{i i}=P_{i i} Q_{i}^{-1} R_{i i}$ and are in bicoprime fractional representation for $i=1,2$. By hypothesis, they have disjoint poles for $i=1$ and $i=2$ so that $\operatorname{det} Q_{1}$ and $\operatorname{det} Q_{2}$ are coprime in $\mathbf{S}$, which implies that both sides in (6) must be matrices over $\mathbf{S}$. In other words, we can write

$$
Q_{i}=\hat{D}_{j} \hat{C}_{j}, D_{j j}=\hat{D}_{j} U_{j}, C_{j j}=V_{j} \hat{C}_{j}
$$

for suitable matrices over $\mathbf{S}$ and for $i, j=1,2, i \neq j$ such that $V_{1} U_{1}=V_{2} U_{2}$. We now show that $U_{j}$ and $V_{j}$ are actually unimodular matirices as a consequence of hypothesis $\mathbf{A}$. In fact, by $\mathbf{A}$, we have $\operatorname{det} Q \simeq \operatorname{det} Q_{1} \operatorname{det} Q_{2}$, which gives $\operatorname{det} Q_{i} \simeq \operatorname{det} C_{j} \operatorname{det} D_{j}=$ for $i, j=1,2$ and $i \neq j$. But then, using (5) and (7), $\operatorname{det} \hat{C}_{j} \operatorname{det} \hat{D}_{j} \simeq$ $\operatorname{det} \hat{C}_{j} \operatorname{det} \hat{D}_{j} \operatorname{det} U_{j} \operatorname{det} V_{j}$, which gives $\operatorname{det} U_{j} \operatorname{det} V_{j} \simeq 1$, i.e., $U_{j}, V_{j}$ are indeed unimodular matrices for $j=1,2$. Hence, by (7), $Q_{i}=D_{j j}\left(V_{j} U_{j}\right)^{-1} C_{j j}, i, j=1,2, i \neq j$. We can now redefine
$D_{i i} \leftrightarrow D_{i i}\left(V_{i} U_{i}\right)^{-1}$ for $i=1,2$ so that still $D_{1}^{-1} D_{2}=D_{22} D_{11}^{-1}$ by $V_{1} U_{1}=V_{2} U_{2}$ and $Q_{i}=D_{j j} C_{j j}$ for $i \neq j$.

Supposing (3) exists, let us express $Z_{11}$ and $Z_{22}$ in left and right coprime fractional representations. Let

$$
\begin{aligned}
D_{11}^{-1} R_{22} & =\tilde{R}_{2} \tilde{D}_{1}^{-1}, D_{22}^{-1} R_{11}=\tilde{R}_{1} \tilde{D}_{2}^{-1} \\
P_{11} C_{22}^{-1} & =\tilde{C}_{2}^{-1} \tilde{P}_{1}, P_{22} C_{11}^{-1}=\tilde{C}_{1}^{-1} \tilde{P}_{2}
\end{aligned}
$$

for left coprime matrices $\left(\tilde{C}_{i}, \tilde{P}_{j}\right)$ and right coprime matrices $\left(\tilde{R}_{j}, \tilde{D}_{i}\right), i \neq j$. Also, let

$$
\begin{aligned}
& \tilde{P}_{1} D_{22}^{-1}=\hat{D}_{2}^{-1} \hat{P}_{1}, \tilde{P}_{2} D_{11}^{-1}=\hat{D}_{1}^{-1} \hat{P}_{2} \\
& C_{22}^{-1} \tilde{R}_{1}=\hat{R}_{1} \hat{C}_{2}^{-1}, C_{11}^{-1} \tilde{R}_{2}=\hat{R}_{2} \hat{C}_{1}^{-1}
\end{aligned}
$$

where, for $i \neq j,\left(\hat{D}_{i}, \hat{P}_{j}\right)$ are left coprime and $\left(\hat{R}_{i}, \hat{C}_{j}\right)$ are right coprime. It follows, by various coprimeness conditions, that for $i, j=$ $1,2, i \neq j$

$$
Z_{i i}=\left(\hat{D}_{j} \tilde{C}_{j}\right)^{-1} \hat{P}_{i} R_{i i}=P_{i i} \hat{R}_{i}\left(\tilde{D}_{j} \hat{C}_{j}\right)^{-1}
$$

are right and left coprime fractions over $\mathbf{S}$, respectively.

Theorem 1: If $\mathbf{A}$ holds and if unstable poles of the diagonal subsystems are disjoint, then $Z$ has no unstable decentralized fixed modes.

Proof: By Lemma 1, a bicoprime fraction (3), where $Q=D_{1} D_{22} C_{22} C_{21}$ is say $r \times r$, exists. By [1] and by [4, Ch. 7] $Z$ is free of unstable decentralized fixed modes if and only if the matrices

$$
\left[\begin{array}{cc}
D_{1} D_{22} C_{11} C_{2} & D_{2} R_{22} \\
P_{11} C_{1} & 0
\end{array}\right],\left[\begin{array}{cc}
D_{2} D_{11} C_{22} C_{1} & D_{1} R_{11} \\
P_{22} C_{2} & 0
\end{array}\right]
$$

are complete over $\mathbf{S}$, i.e., their first $r$ invariant factors are units of $\mathbf{S}$. Using coprimeness of (4) and (8), it follows that the first matrix is complete if and only if

$$
\left[\begin{array}{cc}
D_{22} C_{11} & D_{22} \tilde{R}_{2} \\
\tilde{P}_{1} C_{11} & 0
\end{array}\right]
$$

is complete. This is because the first matrix in (11) and (12) can be shown to have the same invariant factors over $\mathbf{S}$. By hypothesis, (det $D_{22}$, det $C_{11}$ ) are coprime since their unstable zeros belong to poles of different diagonal subsystems. By [4, Cor. (2.8)], the matrices $\left(D_{22}, C_{11}\right)$ are skew prime over $\mathbf{S}$, i.e., there are matrices $\hat{D}_{22}, \hat{C}_{11}$ such that $D_{22} C_{11}=\hat{C}_{11} \hat{D}_{22}$ with $\left(C_{11}, \hat{D}_{22}\right)$ right and $\left(D_{22}, \hat{C}_{11}\right)$ left coprime over $\mathbf{S}$. Now, (9) implies that (12) is complete if and only if $\left[\begin{array}{cc}I & \hat{D}_{22} \hat{R}_{2} \\ \hat{P}_{1} \hat{C}_{11} & 0\end{array}\right]$ is complete. But, this matrix is obviously complete over $\mathbf{S}$ as its first $r$ invariant factors are unity. By similar arguments, the second matrix in (11) is also complete and the result follows.

Remark 1: When A holds and the diagonal subsystems have common unstable poles, the unstable decentralized fixed modes seem to arise from the common diagonal subsytem poles and (central) unstable zeros. It is easy to see this, using the results in [5], for the special case when $Z$ is $2 \times 2$. Let $Z=\left[\left(n_{i j}\right) /\left(m_{i j}\right)\right]=M^{-1} N$, where $\left(m_{i j}, n_{i j}\right), i, j=1,2$ are coprime elements in $\mathbf{S}$ and $(M, N)$ are $2 \times 2$ left coprime matrices over $\mathbf{S}$. Then, the common unstable zeros of $\left\{m_{11}, m_{22}\right.$, det $\left.N\right\}$ are precisely the unstable decentralized fixed modes of $Z$.

Since, plants free of unstable decentralized fixed modes can be stabilized by a decentralized controller, [13], Theorem 1 gives that plants satisfying assumption $\mathbf{A}$ and having disjoint unstable poles in the diagonal admit decentralized stabilizing controllers. We show now that, for such plants, we can do much better.

Theorem 2: Suppose $\mathbf{A}$ holds and the diagonal subsystems $Z_{11}$ and $Z_{22}$ have their unstable poles disjoint. Then, there exists a decentralized controller simultaneously stabilizing $Z_{d}$ and $Z$. 
Proof: By Lemma 1, we have the fraction (3). We now establish that there exist controllers $Z_{c i}:=L_{i} K_{i}^{-1}$ and matrices $\tilde{L}_{i}$, for $i=$ 1,2 , satisfying the following conditions simultaneously:

$$
\begin{aligned}
& \tilde{C}_{j} K_{i} \hat{D}_{j}+\tilde{P}_{i} \tilde{R}_{i} \tilde{L}_{i}=I \quad i, j=1,2, i \neq j \\
& U:=I-\tilde{P}_{1} \tilde{R}_{2} \tilde{L}_{2} \tilde{P}_{2} \tilde{R}_{1} \tilde{L}_{1} \text { is unimodular, and } \\
& L_{i}:=\tilde{D}_{j} \tilde{L}_{i} \hat{D}_{j}^{-1}, i, j=1,2, i \neq j .
\end{aligned}
$$

are matrices over $\mathbf{S}$. We can then rewrite (13) as

$$
\hat{D}_{j} \tilde{C}_{j} K_{i}+\hat{P}_{i} R_{i i} L_{i}=I, i \neq j .
$$

by (15) so that $Z_{c i}$ is a stabilizing controller for $Z_{i i}$ for $i=1,2$. Moreover, the closed-loop denominator matrix attained by $Z_{c i}$, i.e.,

$$
Q_{11}=\left[\begin{array}{ccc}
D_{1} D_{22} C_{22} C_{1} & D_{1} R_{11} L_{1} & D_{2} R_{22} L_{2} \\
-P_{11} C_{1} & K_{1} & 0 \\
-P_{22} C_{2} & 0 & K_{2}
\end{array}\right]
$$

satisfies, $\operatorname{diag}\left\{I, \tilde{C}_{2}, \tilde{C}_{1}\right\} Q_{11} \operatorname{diag}\left\{I, \hat{D}_{2}, \hat{D}_{1}\right\}=$

$$
\left[\begin{array}{ccc}
D_{1} D_{22} & 0 & 0 \\
-\tilde{P}_{1} & I & 0 \\
-\tilde{P}_{2} & 0 & I
\end{array}\right]\left[\begin{array}{ccc}
I & \tilde{R}_{1} \tilde{L}_{1} & \tilde{R}_{2} \tilde{L}_{2} \\
0 & I & \tilde{P}_{1} \tilde{R}_{2} \tilde{L}_{2} \\
0 & \tilde{P}_{2} \tilde{R}_{1} \tilde{L}_{1} & I
\end{array}\right] \Lambda
$$

with $\Lambda:=\operatorname{diag}\left\{C_{22} C_{1}, I, I\right\}$, where (8), (9), and (13) are employed. Since $\operatorname{det}\left(D_{1} D_{22}\right)=\operatorname{det} \hat{D}_{1} \operatorname{det} \hat{D}_{2}$ and $\operatorname{det}\left(C_{22} C_{1}\right)=$ det $\tilde{C}_{1}$ det $\tilde{C}_{2}$, it follows that $\operatorname{det} Q_{11}$ is equal to the determinant of the middle matrix, or

$$
\operatorname{det} Q_{11}=\operatorname{det}\left(I-\tilde{P}_{1} \tilde{R}_{2} \tilde{L}_{2} \tilde{P}_{2} \tilde{R}_{1} \tilde{L}_{1}\right)
$$

which is a unit by (14). Therefore, $\operatorname{diag}\left\{Z_{c 1}, Z_{c 2}\right\}$ stabilizes the diagonal part $Z_{d}$ as well as the overall system $Z$.

We now prove the italicized statement above to complete the proof. Let controllers in right coprime fraction $L_{0 i} K_{0 i}^{-1}$ satisfy

$$
\hat{D}_{j} \tilde{C}_{j} K_{0 i}+\hat{P}_{i} R_{i i} L_{0 i}=I, \quad i, j=1,2, \quad i \neq j .
$$

Such controllers exist since, by $(10),\left(\hat{D}_{j} \tilde{C}_{j}, \hat{P}_{i} R_{i i}\right)$ are left coprime for $i, j=1,2, i \neq j$. Let $d_{i}:=\operatorname{det} \hat{D}_{i} \simeq \operatorname{det} D_{i}$ and $c_{i}:=$ $\operatorname{det} \tilde{C}_{i} \simeq \operatorname{det} C_{i}$ for $i=1,2$ and note that $\left(c_{2} d_{2}, \hat{D}_{1} \tilde{C}_{1}\right)$ is left coprime. We can hence choose $L_{02}$ such that $c_{2} d_{2}$ divides it, i.e., $L_{02}=$ $c_{2} d_{2} H_{02}$ for some matrix $H_{02}$ over $\mathbf{S}$. By (18), $\tilde{L}_{i 0}:=\tilde{D}_{i}^{-1} L_{i 0} \hat{D}_{i}$ is a matrix over $\mathbf{S}$ and satisfies

$$
\tilde{C}_{j} K_{0 i} \hat{D}_{j}+\tilde{P}_{i} \tilde{R}_{i} \tilde{L}_{0 i}=I, \quad i, j=1,2, \quad i \neq j .
$$

Consider, for arbitrary $X_{i}$ over $\mathbf{S}$ and for $i, j=1,2, i \neq j$,

$$
K_{i}=K_{0 i}-P_{i} \hat{R}_{i} X_{i} \quad \tilde{L}_{i}=\tilde{L}_{0 i}+\hat{C}_{j} X_{i} \hat{D}_{j}
$$

which clearly still satisfy (19), i.e., $\tilde{C}_{j} K_{i} \hat{D}_{j}+\tilde{P}_{i} \tilde{R}_{i} \tilde{L}_{i}=I, i, j=$ $1,2, i \neq j$. Also note that, if $X_{2}=c_{2} d_{2} Y_{2}$ for some $Y_{2}$, then $\tilde{L}_{2}=$ $c_{2} d_{2} \tilde{H}_{2}$ for some $H_{2}$ over $\mathbf{S}$. It can be directly verified that the condition (15) is also satisfied for any $X_{1}$ and $Y_{2}$. We now choose $Y_{2}$ and $X_{1}$ to ensure that $\tilde{L}_{i} K_{i}^{-1}$ satisfy the condition (14). Let us first note that $Y_{2}$ can be fixed so as to make

$$
M:=I-\tilde{P}_{1} \tilde{R}_{2} \tilde{L}_{2} \tilde{P}_{2} \tilde{R}_{1} \tilde{L}_{01}
$$

nonsingular. In fact, let $y$ be such that $Y_{2}:=y \hat{C}_{2}^{-1} \tilde{L}_{02} \hat{D}_{2}^{-1}$ is over $\mathbf{S}$ and at some real number $\alpha, y(\alpha)=1$. (Choose, for instance, $\alpha$ to be any real number that is neither a zero of $d_{2}$ nor $c_{2}$ and define $y(s)=\left(c_{2}(s) d_{2}(s)\right) /\left(c_{2}(\alpha) d_{2}(\alpha)\right.$.) Now, we can write $M=I-(1-y) \tilde{P}_{1} \tilde{R}_{2} \tilde{L}_{20} \tilde{P}_{2} \tilde{R}_{1} \tilde{L}_{01}$ which satisfies $M(\alpha)=I$ so that $M$ is nonsingular. With $Y_{2}$, and hence $X_{2}$, so fixed we next choose $X_{1}$ such that $U=M-\tilde{P}_{1} \tilde{R}_{2} \tilde{L}_{2} \tilde{P}_{2} \tilde{R}_{1} \hat{C}_{2} X_{1} \hat{D}_{2}=$
$I \quad-\quad \tilde{P}_{1} \tilde{R}_{2} \tilde{H}_{2} \tilde{P}_{2} \tilde{R}_{1} c_{2} \tilde{L}_{01} d_{2}-\tilde{P}_{1} \tilde{R}_{2} \tilde{H}_{2} \tilde{P}_{2} \tilde{R}_{1} c_{2} \hat{C}_{2} d_{2} X_{1} \hat{D}_{2} \quad$ is unimodular, where the last expression is by $\tilde{L}_{2}=c_{2} d_{2} \tilde{H}_{2}$. Let $T:=\hat{D}_{2} \tilde{P}_{1} \tilde{R}_{2} \tilde{H}_{2} \tilde{P}_{2} \tilde{R}_{1} c_{2}, G:=T \hat{C}_{2} d_{2}$. We consider $\hat{U}:=\hat{D}_{2} U \hat{D}_{2}^{-1}$, which is $\hat{U}=I-T \hat{L}_{01} \operatorname{adj} \hat{D}_{2}-G X_{1}$. Clearly, $U$ is unimodular if and only if $\hat{U}$ is. Referring to [12, Cor. 5.3.6 and Th. 4.4.2], we need only show that at the unstable zeros of the smallest invariant factor of $G$, i.e., at the unstable zeros of $\operatorname{sif}(G)$, $\operatorname{det}\left(I-T \tilde{L}_{01}\right.$ adj $\left.\hat{D}_{2}\right)$ has constant sign. For any square nonsingular matrix $B$ and any rectangular $A$, it is easy to see that $\operatorname{sif}(\mathrm{AB})$ divides det $B \operatorname{sif}(A)$. Thus, $\operatorname{sif}(G) \operatorname{divides} c_{2} d_{2} \operatorname{sif}(T)$. Now, if any complex number $s_{0}$ in the right half plane is either a zero of $c_{2}$ or a zero of $\operatorname{sif}(T)$, then $\operatorname{det}\left[I-T\left(s_{0}\right) \tilde{L}_{01}\left(s_{0}\right) \operatorname{adj} \hat{D}_{2}\left(s_{0}\right)\right]=\operatorname{det} I=1$. Also, if $s_{0}$ is an unstable zero of $d_{2}$, then

$$
\begin{aligned}
\operatorname{det}\left[I-T\left(s_{0}\right) \tilde{L}_{01}\left(s_{0}\right)\right. & \left.\operatorname{adj} \hat{D}_{2}\left(s_{0}\right)\right] \\
& =\operatorname{det}\left\{I-\left[\operatorname{adj} \hat{D}_{2}\left(s_{0}\right)\right] T\left(s_{0}\right) \tilde{L}_{01}\left(s_{0}\right)\right\}=1
\end{aligned}
$$

since $d_{2}$ divides $\left(\operatorname{adj} \hat{D}_{2}\right) T$. Therefore, at all unstable zeros of $c_{2} d_{2} \operatorname{sif}(T)$, and of $\operatorname{sif}(G), \operatorname{det}\left(I-T \tilde{L}_{01}\right.$ adj $\left.\hat{D}_{2}\right)$ has constant sign so that $\hat{U}$ and $U$ can be made unimodular by a suitable $X_{1}$.

Remark 2: If $\mathbf{A}$ holds but $Z_{11}$ and $Z_{22}$ have common unstable poles, then one can show, in the $2 \times 2$ case of Remark 1 , that $Z_{d}$ and $Z$ can be simultaneously stabilized if and only if there are an even number of unstable zeros of $n_{11} n_{22} \operatorname{det} N$ between every pair of real unstable zeros of $g c f\left\{m_{11}, m_{22}\right\}$. This condition corresponds to $\left(g c f\left\{m_{11}, m_{22}\right\}, n_{11} n_{22} \operatorname{det} N\right)$ being coprime and having the parity interlacing property. In the multivariable case, one may thus expect that $Z_{d}$ and $Z$ can be simultaneously stabilized if and only if there are an even number of elements of the set $\left\{s: Z_{11}(s)=0\right.$ or $Z_{22}(s)=0$ or $\left.Z(s)=0\right\}$ between every pair of common real, unstable diagonal subsytem poles. As a consequence, if det $N$ is identically zero, then the lack of unstable common poles of $Z_{11}$ and $Z_{22}$ becomes a necessary condition for solution of the problem.

The construction in Theorem 2 of local stabilizing controllers achieving global stability can be summarized as follows.

i) Obtain the fractional representation (3) for $Z$ following the procedure of Lemma 1.

ii) Determine initial stabilizing local controllers $L_{0 i} K_{0 i}^{-1}$ satisfying (18). Make sure that $c_{2} d_{2}$ divides $L_{02}$.

iii) Determine $Y_{2}$ such that $M$ of (21) is nonsingular. Let $X_{2}:=$ $c_{2} d_{2} Y_{2}$.

iv) Determine $X_{1}$ such that $\hat{U}$ is unimodular using [12, Cor. 5.3.6].

v) Compute $L_{i} K_{i}^{-1}$ according to (20) and (15), where $X_{1}$ and $X_{2}$ are substituted from ii) and iii).

The following simple example illustrates this procedure.

Example: Consider

$$
\begin{aligned}
Z & =\left[\begin{array}{cc}
\frac{1}{2(s-1)} & -\frac{1}{2 s} \\
\frac{1}{2(s-1)} & \frac{1}{2 s}
\end{array}\right] \\
& =\left[\begin{array}{cc}
\frac{s}{s+1} & -\frac{s}{s+1} \\
-\frac{1}{s+1} & \frac{2 s-1}{s+1}
\end{array}\right]^{-1}\left[\begin{array}{cc}
0 & -\frac{1}{s+1} \\
\frac{1}{s+1} & \frac{1}{s+1}
\end{array}\right]
\end{aligned}
$$

in which

$$
\begin{aligned}
P_{1} & =\left[\begin{array}{ll}
1 & 0
\end{array}\right] \quad P_{2}=\left[\begin{array}{ll}
0 & 1
\end{array}\right] \quad R_{1}=\left[\begin{array}{ll}
0 & \frac{1}{s+1}
\end{array}\right]^{\prime} \\
R_{2} & =\left[-\frac{1}{s+1} \frac{1}{s+1}\right]^{\prime}
\end{aligned}
$$

and $Q$ is the denominator matrix shown previously. 
i) We obtain (3) by $C_{1}=C_{2}=C_{11}=C_{22}=I_{2}$ and by

$$
\begin{aligned}
D_{1} & =\left[\begin{array}{cc}
\frac{s}{s+1} & 0 \\
0 & 1
\end{array}\right] \quad D_{22}=\left[\begin{array}{cc}
1 & -1 \\
-\frac{1}{s+1} & \frac{2 s-1}{s+1}
\end{array}\right] \\
R_{11} & =\left[\begin{array}{c}
0 \\
\frac{1}{s+1}
\end{array}\right], D_{2}=\left[\begin{array}{cc}
1 & 0 \\
-1 & \frac{s-1}{s+1}
\end{array}\right] \\
D_{11} & =\left[\begin{array}{cc}
\frac{s}{s+1} & -\frac{s}{s+1} \\
1 & 1
\end{array}\right] \quad R_{22}=\left[\begin{array}{c}
-\frac{1}{s+1} \\
0
\end{array}\right] .
\end{aligned}
$$

It can be checked that $D_{1} D_{22}=D_{2} D_{11}, Q=D_{1} D_{22} C_{22} C_{1}$ and (3) is obtained. Let us alo identify left and right coprime fractions for $Z_{11}$ and $Z_{22}$. We have $D_{22}^{-1} R_{11}=\tilde{R}_{1} \tilde{D}_{2}^{-1}$, and $D_{11}^{-1} R_{22}=\tilde{R}_{2} \tilde{D}_{1}^{-1}$, where

$$
\begin{aligned}
& \tilde{R}_{1}=\left[\frac{1}{2(s+1)}\right] \quad \tilde{D}_{2}=\frac{s-1}{s+1} \\
& \tilde{R}_{2}=\left[\begin{array}{c}
-\frac{1}{2(s+1)} \\
\frac{1}{2(s+1)}
\end{array}\right] \quad \tilde{D}_{1}=\frac{s}{s+1} .
\end{aligned}
$$

Also, $P_{i i}=\tilde{P}_{i}=P_{i}, i=1,2$ and $C_{i i}=\tilde{C}_{i}=C_{i}=I, i=$ 1,2. Moreover, $\tilde{P}_{1} D_{22}^{-1}=\hat{D}_{2}^{-1} \hat{P}_{1}$ and $\tilde{P}_{2} D_{11}^{-1}=\hat{D}_{1}^{-1} \hat{P}_{2}$, where $\hat{D}_{1}=s /(s+1)$

$$
\hat{D}_{2}=\frac{s-1}{s+1} \quad \hat{P}_{1}=\left[\frac{2 s-1}{2(s+1)} \frac{1}{2}\right] \quad \hat{P}_{2}=\left[-\frac{1}{2} \frac{s}{2(s+1)}\right] \text {. }
$$

ii) Initial stabilizing controllers for

$$
\begin{aligned}
& Z_{11}=\left(\hat{D}_{2} \tilde{C}_{2}\right)^{-1} \hat{P}_{1} \quad R_{11}=\left(\frac{s-1}{s+1}\right)^{-1} \frac{1}{2(s+1)} \\
& Z_{22}=\left(\hat{D}_{1} \tilde{C}_{1}\right)^{-1} \quad \hat{P}_{2} R_{22}=\left(\frac{s}{s+1}\right)^{-1} \frac{1}{2(s+1)}
\end{aligned}
$$

are easily computed (in this simple case by inspection) as $L_{0 i} K_{0 i}^{-1}, i=1,2$ with $K_{01}=1, \tilde{L}_{01}=L_{01}=4, K_{02}=$ $(s+3) /(s+1), \tilde{L}_{02}=L_{02}=-2(s-1) /(s+1)$, where $c_{2} d_{2}=(s-1) /(s+1)$ is a factor of $L_{02}$.

iii) Here, on letting $X_{2}=(s-1) /(s+1) Y_{2}$, we have $M=$ $1+(1) /\left((s+1)^{2}\right)\left[-2(s-1) /(s+1)+(s(s-1)) /\left((s+1)^{2}\right) Y_{2}\right]$ nonsingular for the choice $Y_{2}=0$. Thus, $U=1-(s-$ $1) /\left(2(s+1)^{3}\right)\left(4+(s-1) /(s+1) X_{1}\right)$.

iv) Note that when evaluated at the two unstable blocking zeros $s=$ 1 and $s=\infty$ of the coefficient of $X_{1}, 1-(2(s-1)) /\left((s+1)^{3}\right)$ is equal to 1 . Restricting $X_{1}$ to be of first order, it can be computed, using e.g., root-locus, that $U$ is unimodular for $X_{1}=$ $0.286(s+5) /(s+0.1)$ with its zeros at $\{-3.0699,-0.1719 \pm$ $0.7416 i,-0.3432 \pm 0.6666 i\}$.

v) Fixing $X_{1}$ as in iv) and $X_{2}=0$, we obtain that

$$
\begin{aligned}
Z_{c} & =\left[\begin{array}{cc}
\frac{4(s+1)(s+0.1)+0.286(s-1)(s+5)}{(s+1)(s+0.1)-0.143(s+5)} & 0 \\
0 & -2 \frac{s-1}{s+3}
\end{array}\right] \\
& =\left[\begin{array}{cc}
\frac{4.286 s^{2}+5.544 s-1.030}{s^{2}+0.957 s-0.615} & 0 \\
0 & -2 \frac{s-1}{s+3}
\end{array}\right]
\end{aligned}
$$

simultaneously stabilizes $Z_{d}$ and $Z$.

Note that the construction of Theorem 2 is not necessarily efficient, i.e., it may involve more computations or yield higher order conrollers than necessary in some cases. In fact, for our example in which diagonal subsystems are scalar, the second controller need not contain the unstable zero at $s=1$ and it is easy to check that $\operatorname{diag}\{4,2\}$ is another solution to the problem.

\section{CONCLUSION}

We have shown that the assumption $\mathbf{A}$ is a crucial one for stabilizing a system by stabilizing its main diagonal subsystems and somewhat trivializes the existence of a solution: $\mathbf{A}$ by itself ensures a solution to exist and no extra conditions such as diagonal dominance need be imposed. This has been established in Theorem 2 for the case in which the unstable poles of the diagonal subsytems are disjoint. In the general case, when diagonal subsystems have some common unstable poles, a similar result is expected. If $Z$ is $2 \times 2$, for instance, one can show that it is possible to simultaneously stabilize $Z_{d}$ and $Z$ if and only if there are an even number of zeros of $Z_{11} Z_{22} Z$ between every pair of real, unstable, and common diagonal subsystem poles. Extension of this result to multivariable case is currently under investigation. In closing, we should mention that when stabilization is not the only concern and other design specifications are present, the diagonal dominance property is very useful as illustrated in [7].

\section{ACKNOWLEDGMENT}

The author would like to thank the reviewers for the challenge of giving a direct proof of Theorem 2 .

\section{REFERENCES}

[1] J. P. Corfmat and A. S. Morse, "Decentralized control of linear multivariable systems," Automatica, vol. 8, pp. 479-485, 1976.

[2] M. Morari and E. Zafiriou, Robust Process Control. Upper Saddle River, NJ: Prentice-Hall, 1989.

[3] Y. Ohta, D. D. Šiljak, and T. Matsumoto, "Decentralized control using quasiblock diagonal dominance of transfer function matrices," IEEE Trans. Autom. Control, vol. AC-31, no. 5, pp. 420-429, May 1986.

[4] A. B. Özgüler, Linear Multichannel Control: A System Matrix Approach. Upper Saddle River, NJ: Prentice-Hall, 1994.

[5] A. B. Özgüler and M. Hiraoğlu, "Implications of a characterization result on strong and reliable decentralized control," in Modeling, Robustness and Sensitivity Reduction in Control Systems. ser. NATO ASI, R. F. Curtain, Ed. New York: Springer-Verlag, 1987, vol. F34, pp. 425-450.

[6] H. H. Rosenbrock, "Design of multivariable control systems using the inverse Nyquist array," in Proc. Inst. Elect. Eng., vol. 116, 1969, pp. 1929-1936.

[7] H. H. Rosenbrock, Computer-Aided Control System Design. London, U.K.: Academic, 1974.

[8] K. A. Ünyelioğlu, "Decentralized blocking zeros in the control of large scale systems," Ph.D. dissertation, Elect. Electron. Eng., Bilkent Univ., Ankara, Turkey, 1992.

[9] K. A. Ünyelioğlu and A. B. Özgüler, "Reliable decentralized stabilization of feedforward and feedback interconnected systems," IEEE Trans. Autom. Control, vol. 37, no. 8, pp. 1119-1132, Aug. 1992.

[10] K. A. Ünyelioğlu, Ü. Özgüner, and A. B. Özgüler, "Decentralized fixed zeros of decentralized control systems," IEEE Trans. Autom. Control, vol. 45, no. 1, pp. 146-151, Jan. 2000.

[11] K. A. Ünyelioğlu, Ü. Özgüner, and A. B. Özgüler, "Measurement of interactions in multivariable systems: Some structural issues,", Ankara, Turkey, Bilkent Univ. Rep., 1993.

[12] M. Vidyasagar, Control System Synthesis: A Factorization Approach. Cambridge, MA: MIT Press, 1985.

[13] S. H. Wang and E. J. Davison, "On the stabilization of decentralized control systems," IEEE Trans. Autom. Control, vol. AC-18, no. 5, pp. 473-478, 1973.

[14] Q.-H. Wu and M. Mansour, "Decentralized robust control using $H^{\infty}$-optimization technique," Inform. Decision Technol., vol. 15, pp. 59-76, 1989.

[15] G. Zames and D. Bensoussan, "Multivariable feedback, sensitivity, and decentralized control," IEEE Trans. Autom. Control, vol. AC-28, no. 11, pp. 1030-1035, 1983. 